\title{
Development of a transparent single-grid-type MSGC based on LCD technology
}

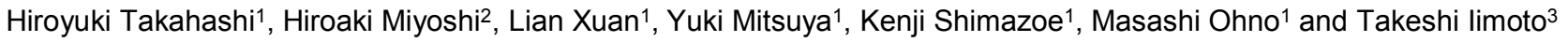 \\ ${ }^{1}$ Departmenf of Nuclear Engineering and Management, Graduate School of Engineering, The University of Tokyo, 7-3-1 Hongo, Bunkyo-ku, \\ Tokyo 113-8656, JAPAN \\ ${ }^{2}$ SHARP Co. Ltd, 22-22 Nagaike-cho, Abeno-ku, Osaka 545-8522, JAPAN \\ ${ }^{3}$ Division for Environment, Health, and Safety, The University of Tokyo, 7-3-1 Hongo, Bunkyo-ku, Tokyo 113-8654, JAPAN
}

\begin{abstract}
We are developing a multi-grid-type MSGC based on the liquid crystal display (LCD) technology, which enables a large area and fine structure. Single-grid-type MSGC using transparent electrodes has been fabricated and successfully operated in several different gas mixtures. The use of LCD technology allows us to integrate some simple electronics using thin film transistors. Such an integrated device is our next target. The successful operation of Single-grid-type MSGC is the very important first step for us.
\end{abstract}

\section{Introduction}

We are working on the use of microfabrication techniques to the radiation detection for almost 20 years. In this period, we studied on MSGC (MicroStrip Gas Chamber), Glass GEM (Gas electron multiplier), and dedicated ASIC (Application Specific Integrated Circuit). Based on our experience in these individual components of present-day's gaseous radiation detectors, we are becoming aware of the importance of more integrated devices. Many principles of the signal readout method are invented but the difficulty of the application of each principle lies in the interface between the sensor part and the readout electronics. New important topics may even exist in the interfacing itself. Now we re-think about the MSGC. People are almost forgetting about the MSGC [1], however, during these decades, big progress has been made in the microfabrication techniques on the glass, in particular, in the field of liquid crystal display (LCD).

Because an essence of the LCD technology is the formation of very precise electrode patterns on a large area glass plate, the LCD technology can readily be applied to the MSGC fabrication. One problem of the conventional MSGC was related to the substrate. The glass substrate isolates the anode and the cathode, however the high resistivity surface of the glass substrate causes a charge-up problem. Although the small-gap MSGC can solve this problem, it cannot operate at high gas gain since the anode and the cathode are too close in this geometry and the surface discharge is unavoidable. We have already shown a new configuration of MSGC using the multi-grid-type electrode structure [2], where the electrodes are not just the anode and the cathode but intermediate electrodes (grids) are inserted in between to stabilize the detector and increase gas gain.

\footnotetext{
${ }^{a}$ Corresponding author: leo@n.t.u-tokyo.ac.jp
}

Also, we demonstrated that the MSGC with transparent electrodes can be operated in an $\mathrm{Ar} / \mathrm{CF}_{4}$ gas mixture. Scintillation light is emitted in this gas mixture [3] in the course of avalanche process, which can be seen through the transparent electrode and the glass substrate. The use of optical signal for position sensing was demonstrated [4]. The LCD technology relies on the transparent electrode such as indium tin oxide (ITO) and indium zinc oxide (IZO), which is favourable for the optical readout. Also, we could integrate some simple electronics with thin film transistors (TFTs) on the glass as already done in most of LCD panels. Thus, in principle, we can integrate all the necessary functions for a gaseous radiation detector with readout electronics on just one glass plate as shown in Figure 1.

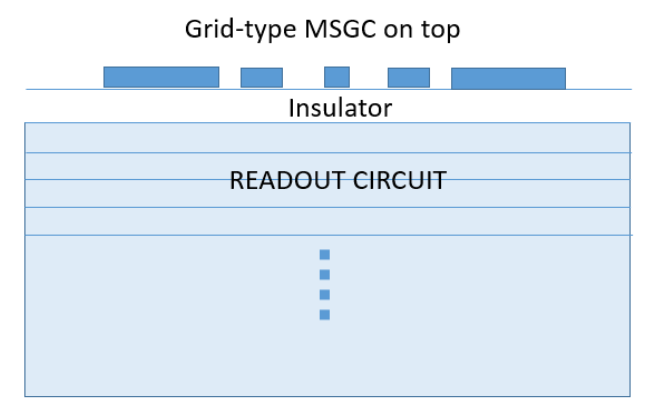

Figure 1. Concept of new generation of MSGC based on LCD technology.

This is definitely compact, efficient, and cost-effective. Besides the optical readout, we can also use induced charge [5], or direct coupling to the MSGC cathode. Based on such an idea, we are intensively working on the development of new generation MSGC. Radiation signals 
can be transferred to the integrated readout circuit by visible photons, induced charges, or direct current (see Figure 2). The simplest readout circuit is just a TFT array with integrated capacitance, which is applied to the flat panel detector for medical applications, however, many other possibilities are being considered in our collaboration team. In particular, the signal readout electronics for the gas detector operated at high voltage often suffers from damages caused by spark events. If we use optical signals as the interface from micropattern gas detector to the readout circuit, we are completely free from the damage problem. Besides this, we could also utilize the charge signal.

\section{HOW TO TRANSFER SIGNAL TO THE READOUT CIRCUIT}

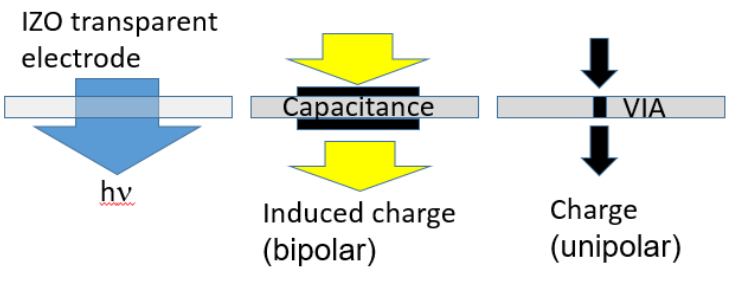

Optical coupling Induced Charge Direct coupling

Figure 2. Three different signal readout schemes through the insulation layer. 'VIA' means electrical connection through the insulation layer.

Based on such an idea, we are fabricating test MSGCs as the first step to the integrated device. We report some of the initial results in this paper.

\section{Design and fabrication of transparent MSGC with a single grid}

We have designed a new single-grid type MSGC (SMSGC) using IZO electrode. Aiming at low energy Xray imaging applications, we selected a fine pitch of 150 $\mu \mathrm{m}$. We adopted a single grid inserted between the anode and the cathode as illustrated in Figure 3. The anode and cathode widths were set to $8 \mu \mathrm{m}$ and $62 \mu \mathrm{m}$, respectively. It is equipped with $20 \mu \mathrm{m}$ wide grid between the anode and the cathode. The grid is biased at an intermediate potential between the anode potential and the cathode potential. The gap widths between neighbouring electrodes are set at $10 \mu \mathrm{m}$, maximizing the surface covered with conductive electrodes. All grid strips are connected together to apply a single bias voltage. Figure 4 shows the fabricated test device. The effective area of the test device was $15 \mathrm{~mm} \times 23 \mathrm{~mm}$.

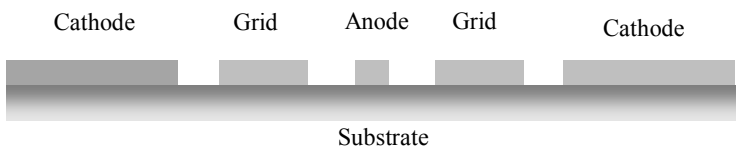

Figure 3. Cross sectional image of the electrode pattern of the designed S-MSGC.

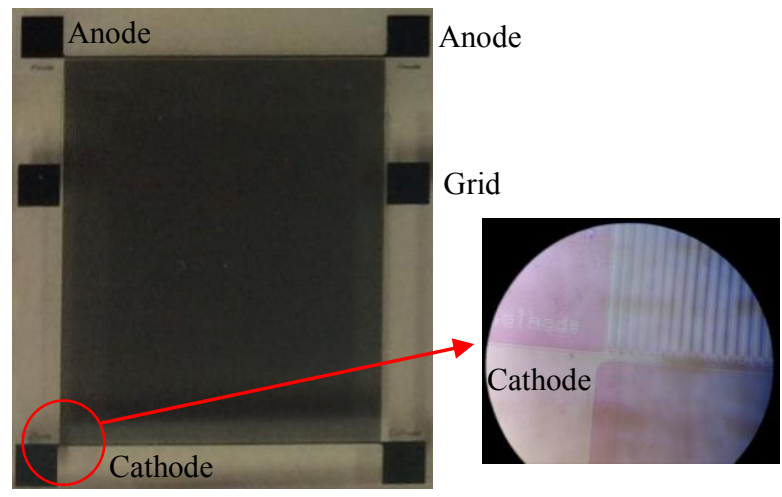

Figure 4. Left: Overall layout image of MSGC electrodes (For visualization purpose, opaque electrodes were used). Right: Microscopy reflection images of IZO electrodes (Near the cathode pad shown in the red circle in the left image). Contrast is adjusted and white part shows the electrode regions.

\section{Test of the transparent S-MSGC}

\subsection{Charge signal measurements}

The S-MSGC was tested under different gas mixtures. We have successfully operated our transparent S-MSGC in $\mathrm{Ar}(90 \%) / \mathrm{CH}_{4}(10 \%), \mathrm{Kr}(90 \%) / \mathrm{CO}_{2}(10 \%)$, and $\operatorname{Ar}(90 \%) / \mathrm{CF}_{4}(10 \%)$. The applied voltage was around $400-550 \mathrm{~V}$ to the anode and $100-250 \mathrm{~V}$ to the grid. The anode strips were connected together and read out by a charge sensitive amplifier. The maximum gas gain was $\sim 500$. A clear photo peak was observed for an ${ }^{55} \mathrm{Fe} \mathrm{X}$-ray source. Figure 5 shows a result from $\mathrm{Kr} / \mathrm{CO}_{2}$ gas, where the anode was biased at $530 \mathrm{~V}$ and the grid voltage was $240 \mathrm{~V}$. Figure 6 shows a result from $\mathrm{Ar} / \mathrm{CF}_{4}$ gas where the anode voltage was $500 \mathrm{~V}$ and the grid voltage was $250 \mathrm{~V}$. This is a reasonable performance for the singlegrid design.

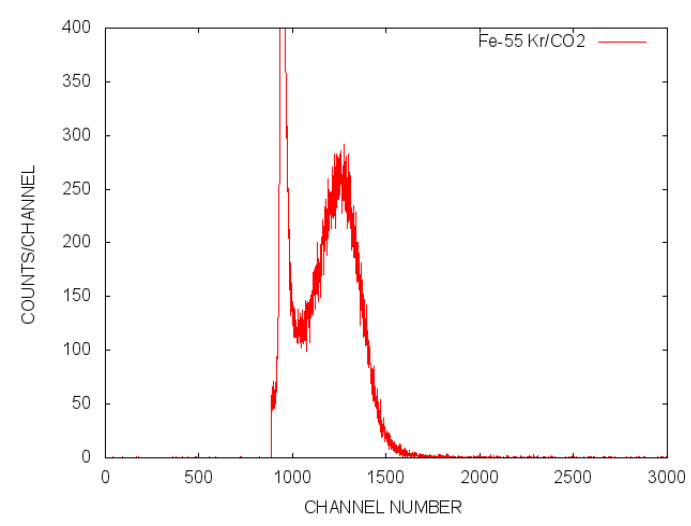

Figure 5. Observed pulse height spectrum for an ${ }^{55} \mathrm{Fe}$ source. The fabricated IZO S-MSGC was operated in a $\mathrm{Kr} / \mathrm{CO}_{2}$ gas mixture. The electric charge was read out from the common anode line. 


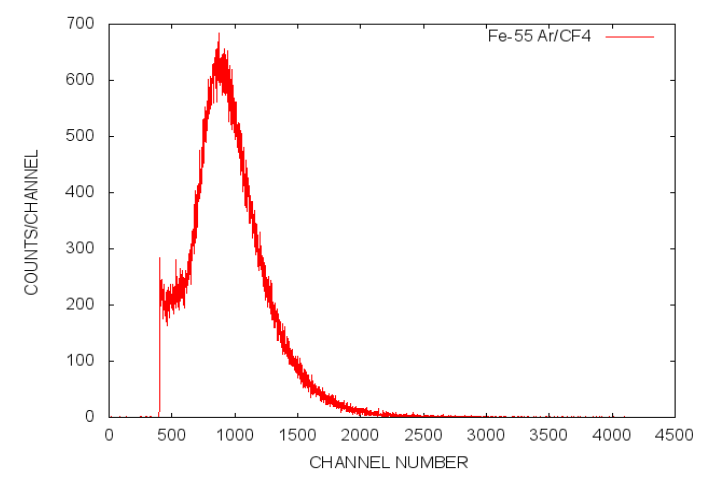

Figure 6. Observed pulse height spectrum for an ${ }^{55} \mathrm{Fe}$ source. The IZO S-MSGC was operated in an $\mathrm{Ar} / \mathrm{CF}_{4}$ gas mixture.

$\mathrm{Kr}$ gas shows a higher mass attenuation coefficient between $17 \mathrm{keV}$ and $30 \mathrm{keV}$ than $\mathrm{Xe}$ gas. Thus, X-ray diagnostics applications are considered with the $\mathrm{Kr}$ gas mixture.

\subsection{Optical signal readout}

Visible light photons are emitted during the avalanche process in $\mathrm{Ar} / \mathrm{CF}_{4}$ gas. The red light at wavelength around $600 \mathrm{~nm}$ is suitable for detection by photodiodes. These photon signals can be used for position sensing and/or pulse height measurement. The cross sectional view of the test setup is shown in Figure 7 and the photograph taken from top is shown in Figure 8 where the IZO electrode pattern is not seen (because of its transparency) and only the Hamamatsu S3590 $10 \mathrm{~mm} \mathrm{x}$ $10 \mathrm{~mm}$ effective area photodiode is visible through IZO S-MSGC.

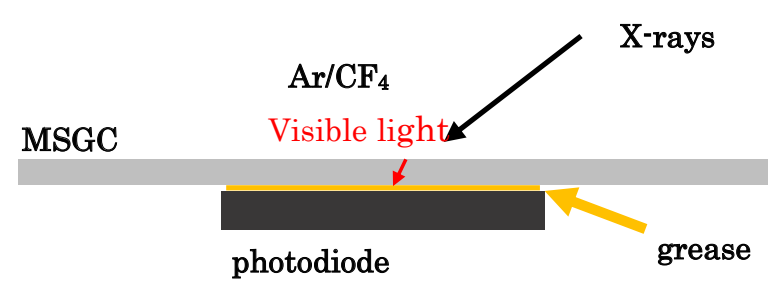

Figure 7. Cross sectional view of the test setup of an optical signal readout

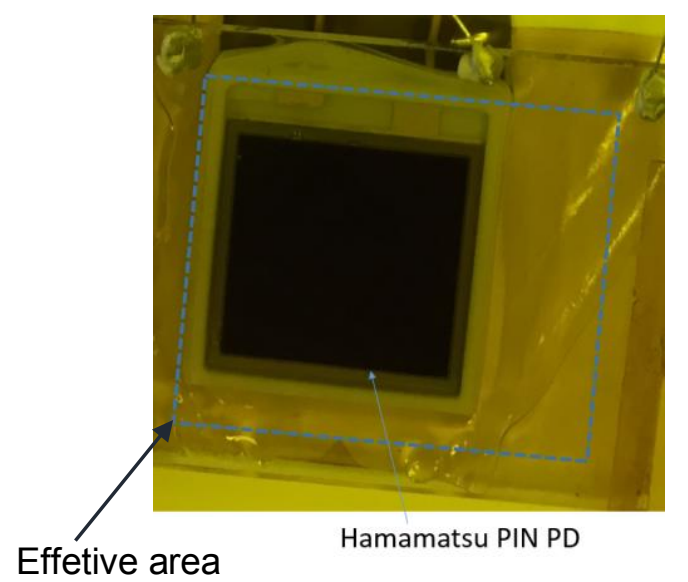

Figure 8. Photograph of IZO S-MSGC with a photodiode.
The anode voltage was $508 \mathrm{~V}$ and the grid voltage was $239 \mathrm{~V}$. The bias voltage of the PIN photodiode was $75 \mathrm{~V}$. Figure 9 shows the pulse height spectra recorded from the PIN photodiode for different radiation sources. BG was obtained without the X-ray source and is considered as the noise level of the measurement system. Due to the large size of the PIN photodiode, the noise level of the preamplifier was quite large. Then, the pulse height spectra do not show photo peaks. However, the pulse height distribution shows longer tail for higher energy Xrays. This implies that larger optical signals are obtained when illuminated by higher energy X-rays, which indicates that the optical signal pulse is due to the radiation detection. This result is encouraging because the readout system is just one large PIN photodiode, whose capacitance is about $40 \mathrm{pF}$. If we utilize a small photodiode such as $150 \mu \mathrm{m} \times 150 \mu \mathrm{m}$ in size, the capacitance is much smaller than that of the large PIN photodiode $(10 \mathrm{~mm} \times 10 \mathrm{~mm}$ effective area). Such photodiodes can be integrated just below the S-MSGC pixel of the same pixel size $(\sim 150 \mu \mathrm{m})$ and this can solve noise problems.

For further optimization of the detector, we are also thinking about the double grid version. Single grid is useful and easy to operate, however, in terms of gas gain, multi-grid design is better. For example, with a test device of two-grid structure, shown in Figure 10, much higher gain was reached. Figure 11 shows a charge spectrum of the two-grid MSGC for ${ }^{55} \mathrm{Fe} \mathrm{X}$-rays. The resolution is significantly improved compared to that of the S-MSGC (Figures 5 and 6). The anode pitch was 80 $\mu \mathrm{m}$ and the anode width was $2 \mu \mathrm{m}$ in this case. Using the latest LCD process, these parameters can be realized.

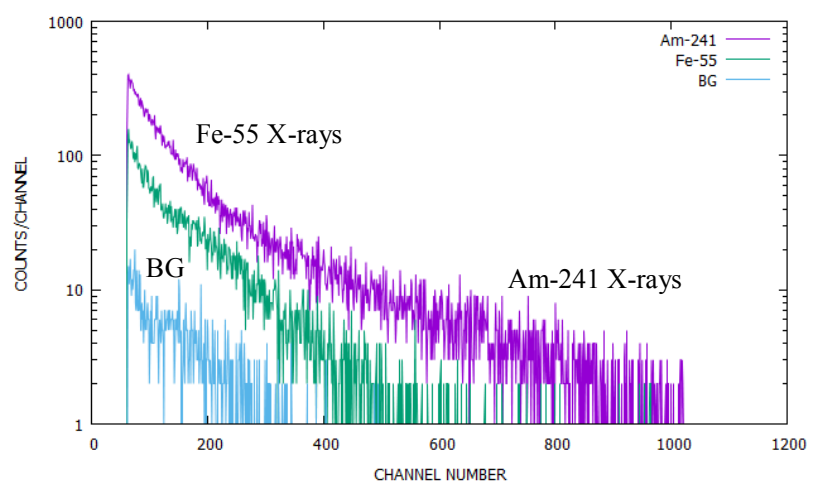

Figure 9. Pulse height spectra obtained with PIN photodiode placed just behind the IZO MSGC (i.e. Optical measurement).

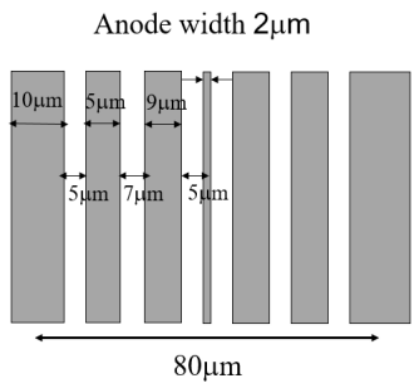

Figure 10. Electrode pattern of a double-grid-type MSGC test device. 


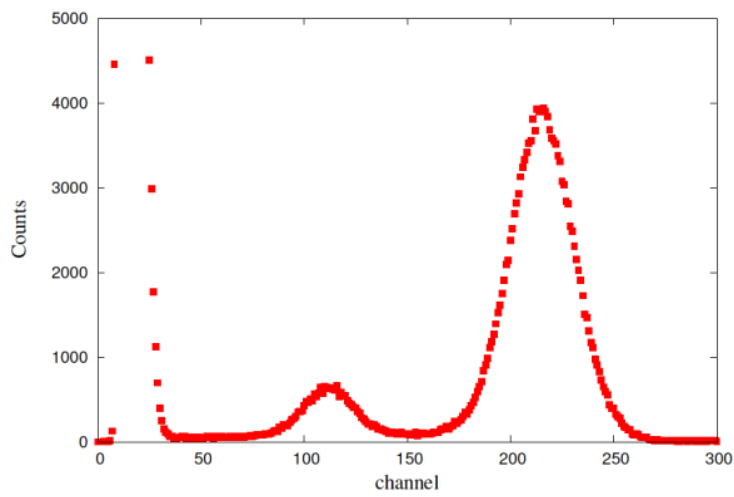

Figure 11. Pulse height spectra of the $80 \mu \mathrm{m}$ anode pitch double-grid-type MSGC obtained with an ${ }^{55} \mathrm{Fe}$ source.

\section{Integration of readout circuits}

To take the full advantage of the LCD technology, integration of the readout circuit based on TFTs (Thin Film Transistors) on the same glass as the MSGC is promising. We are currently investigating the possibilities of such an integrated device and developing a circuit component using TFTs. In this approach, the use of an IGZO (indium gallium zinc oxide) is promising. Figure 12 illustrates the TFT characteristics of IGZO for this approach. It provides a very low leakage current or high resistance, which is useful for charge accumulation device. Therefore, multiplexing readout and integrator circuit will be a primary choice in this device. Also, IGZO is typically operated at tens of volts, which means the device is tolerant to the high voltage when a gaseous detector brings a giant pulse or some discharge, and is promising for the direct coupling to the gas detector signal readout.

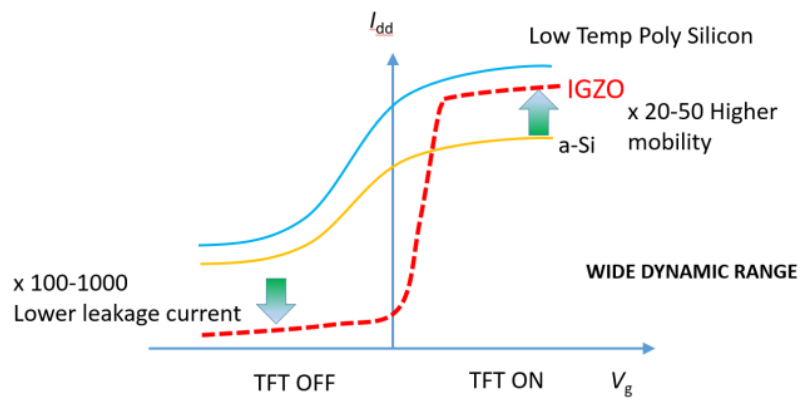

Figure 12. General characteristics of thin film transistor (TFT) available in different process technology.

\section{Conclusions}

We consider X-ray applications and reconsider the use of transparent multi-grid-type MSGC principle. The LCD technology is very promising for the new generation of MSGCs and the transparent IZO electrodes used in this process can provide a new readout scheme with optical photons. We have fabricated a single-grid-type MSGC (S-MSGC) using IZO. The detector has been successfully operated and we also measured optical signals by using a PIN photodiode through the glass substrate with $\mathrm{Ar} / \mathrm{CF}_{4}$ gas. Circuit integration on the same glass substrate is being considered. We anticipate that the highly integrated gaseous detector glass plate may find many applications not only for X-rays but also for many other areas.

\section{References}

1. A. Oed, Nucl. Instr. and Meth. A263, 351 (1988).

2. H. Takahashi, et al., Nucl. Instr. and Meth. A477, 13 (2002).

3. F.A.F. Fraga, et al., Nucl. Instr. and Meth. A 478, 357 (2002).

4. H. Takahashi, et al., Nucl. Instr. and Meth. A623, 123 (2010).

5. K. Fujita, H. Takahashi, et al., Nucl. Instr. and Meth. A580, 1027 (2007). 